\title{
Determination of the beginning the Steady-State for controlled processes in monitoring systems with limited resources
}

\author{
Vladimir Leontiev ${ }^{1, *}$, Alexander Sorokin ${ }^{2}$, and Sergey Saradzhishvili ${ }^{1}$ \\ ${ }^{1}$ Peter the Great St. Petersburg Polytechnic University, Polytechnicheskaya 29, St. Petersburg, \\ 195251, Russian Federation \\ ${ }^{2}$ Saint Petersburg State University of Aerospace Instrumentation (SUAI), Bolshaya Morskaya 67, \\ St.Petersburg, 190000, Russian Federation
}

\begin{abstract}
This paper describes the basic approach for determination of the steady-state conditions of physical processes such as boiling or other phase transitions in control systems with limited resources. In this research, we consider the particular case of a control system that should determine the moment when the water temperature reaches boiling point. In different systems, the accurate determination of the boiling point is important, because it results in other functions such as switching off or transitioning to other states. Hence, we explore the possibility of applying our approach in systems with limited resources, using cheap temperature inertial sensors. During the investigation, we encountered a delay in identifying the boiling point, caused by inertia. These issues demonstrate the need for the further improvement of the suggested approach in this particular case. However, generally our results indicate the applicability of this approach for control systems.
\end{abstract}

\section{Introduction}

Considering the general approaches used for determining steady-state conditions in systems that determine specific time points, which affects decision-making control systems, we consider the particular case of the boiling point.

For such systems, it is important to determine control points accurately. These systems encounter distinctive conditions such as temperature sensor tolerance and inertia. For highly accurate sensors, which could be used in monitoring systems [1,2], a simple temperature measurement could be applicable.

\footnotetext{
*Corresponding author: volodyaleo@gmail.com
} 


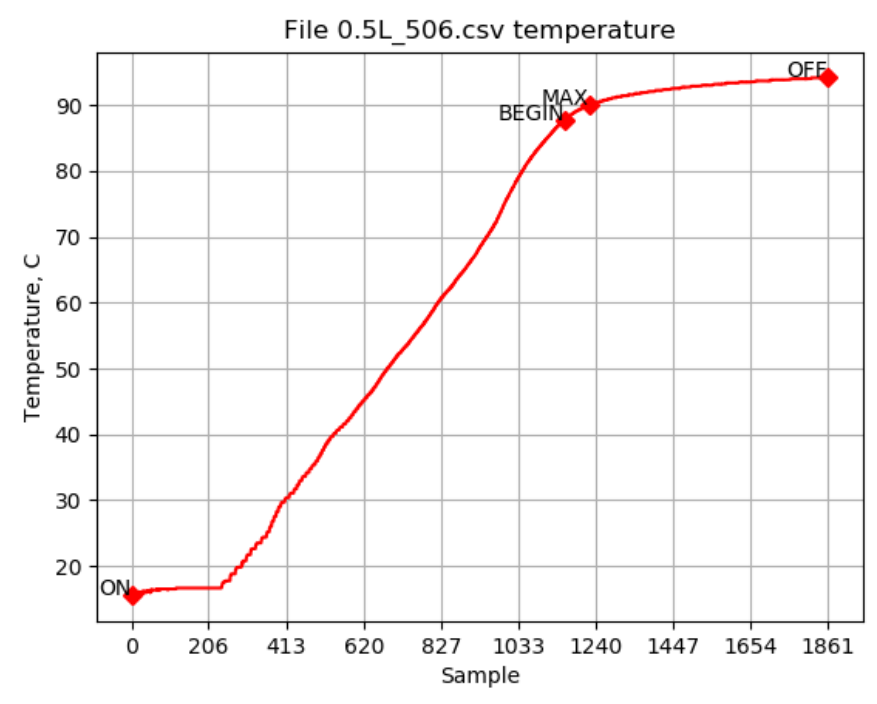

Fig. 1. Simple illustration of a temperature control system.

Figure 1 displays the temperature curve and specific key moments in the boiling process of water: start of the boiling process, moment when the system reaches boiling point, and the switching off point. Here, "ON" refers to the start of the heating process, "Begin" signifies the appearance of rising water bubbles, and "MAX" indicates the switching off point of the heater. Further, "OFF" is the time when the system recognizes the beginning of the steadystate, which means the actual switching off point for the heater.

In this scenario, the control system receives temperature data from sensors, and then determines a boiling point for instigating further actions such as switching off the heater. The accuracy of boiling point determination clearly depends on temperature sensor tolerance. The specific sensor construction, and its positioning, could generate inertia that would affect receiving the actual data for the system. In other words, there is a delay before the system receives the data. This could be a critical point for high-accuracy monitoring systems applied in the automation industry, aerospace, and aviation [1-5].

Considering the example of a system using a simple temperature sensor, which adds an additional variable delay in the determination of specific time points, this resulted in variable time points for the switching phases. This was due to the sensor inertia, which caused inaccuracies in identifying the required time points. Moreover, the amount of inertia varies for different types of sensor. For instance, for an NTC thermistor used as a temperature sensor, the time delay could be $60-80 \mathrm{~s}$ [6-12].

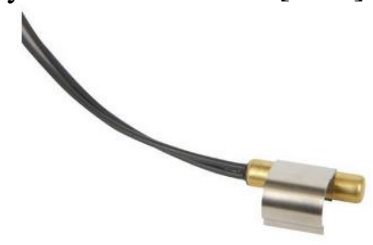

Fig. 2. Typical NTC temperature sensor

Figure 2 shows a typical NTC temperature sensor, and its construction could cause inertia, because of the position of the NTC thermistor in the metal case. However, when the NTC thermistor is set into the metal case during production there is a position tolerance, which is 
not provided in the datasheet. Therefore, when using sensors with unknown inertia, a control system with limited recourses cannot determine the necessary control point accurately. Hence, an approach capable of recognizing the steady-state is needed [15-20].

\section{Methods}

As described previously, in ideal conditions the accurate determination of the boiling point demands a non-inertial sensor, or a sensor with a known inertia induced time delay. However, the idea of using a non-inertial temperature sensor is not possible in reality. Therefore, we consider the process $\mathrm{T}(\mathrm{t})$, which was shown in Fig. 1, and suggest determining the transitional process in the frequency domain.

Our study is based on using an NTC thermistor as a temperature sensor, and here we consider the process of boiling different volumes of water. We considered $0.5,1.5$, and 1.7 $\mathrm{L}$ in our research.

To meet the requirements of accurate time points and limited computational resources, we propose the fast Walsh-Hadamard transform for converting time domain data into the frequency domain.

Hadamard's transformation is not so demanding on computing resources due to the fact that it does not require computationally complex mathematical operations like sine, cosine, exponential and logarithm. It intends to become simple arithmetic operations like multiplication, addition, and subtraction, by using the Walsh function as a basis function. It is also more accurate due to these operations like sine, cosine, and logarithm with a floating point have a rounding error. This fact makes the transformation non-orthogonal. As an additional optimization, you can use fixed-point numbers or reduce calculations to integer operations.

Let us consider the temperature curve as $\mathrm{T}(\mathrm{t})$. The Walsh-Hadamard transform is defined as follows:

$$
y_{n}=\frac{1}{N} \sum_{i=0}^{N-1} T_{i}(t) W A L(n, i), n=1,2, \ldots, N-1
$$

where $\mathrm{T}(\mathrm{t})$ is the temperature curve related to time, $N$ is the total number of samples, and $W A L(n, i)$ is the Walsh-Hadamard transform.

For simplifying the computation we use a simple rectangular window function $D_{n}(t)$ as follows:

$$
y_{n}=\left(\frac{1}{N} \sum_{i=0}^{N-1} T_{i}(t) \cdot W A L(n, i)\right) D_{n}(t), n=1,2, \ldots, N-1
$$

The figure below illustrates several frequency coefficients that were derived after performing equations 1-2. 

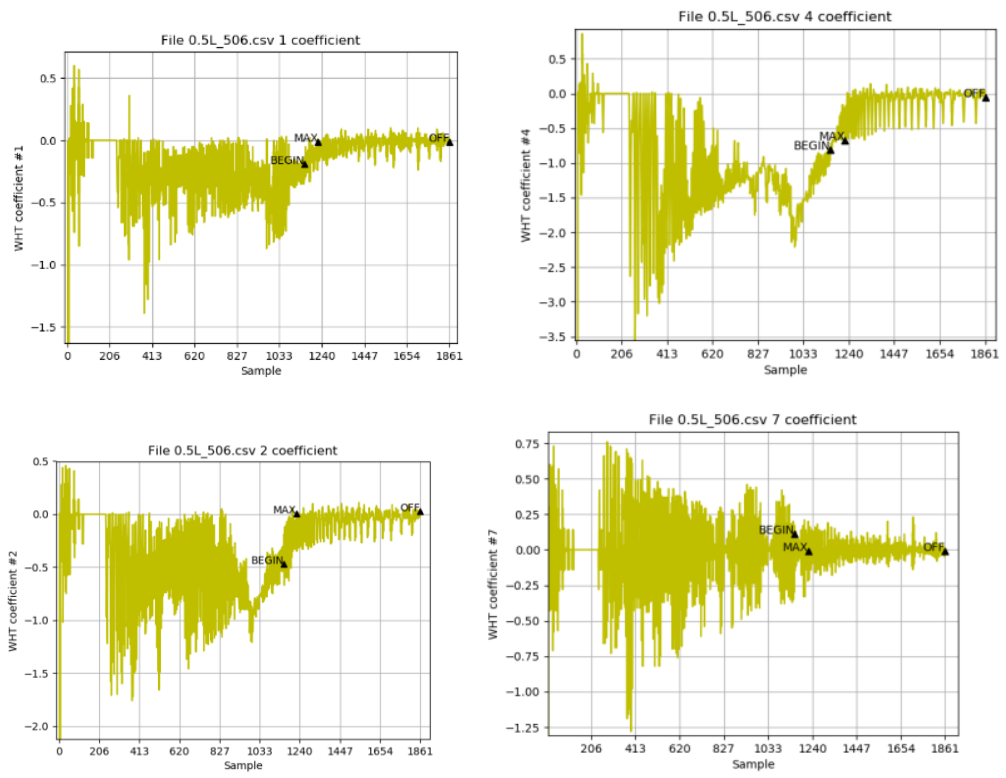

Fig. 3. Graphs showing the eight frequency coefficients derived after the Walsh-Hadamard transform using the window function. After analyzing all the coefficients, only four were taken into consideration. The time span of the window function was defined empirically.

By looking at the eight frequency coefficient functions, we attempted to distinguish the coefficients by identifying distinctive visible signs of steady-state. After the analysis, the fourth coefficient was chosen.

However, the selected coefficient had additional noise. Therefore we attempted to remove the noise by applying a median filter $\operatorname{med}\left(y_{n}\right)$. The results are shown in Fig. 4.

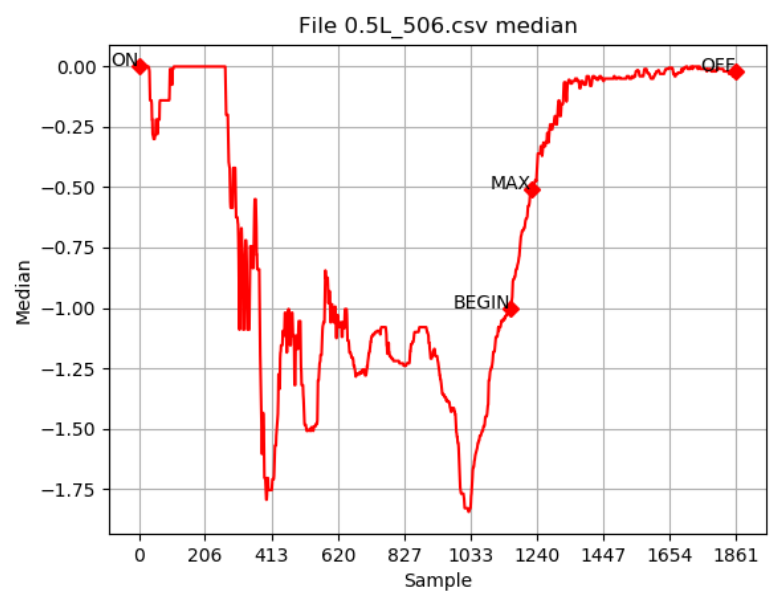

Fig. 4. Removing noise by applying a median filter. Sample step $=0.1 \mathrm{~s}$.

The application of moving average is another possible method suitable for removing noise. 


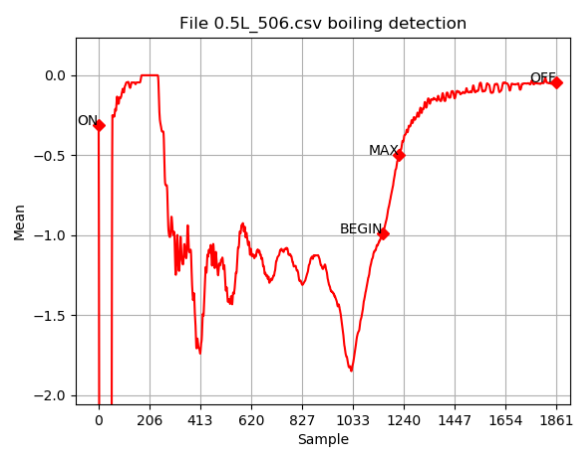

Fig. 5. Removing noise by applying moving average.

An analysis of the results demonstrates that using the moving average appears to be more appropriate because of limited system resources.

The resulting graph shows the necessity of the time threshold and level threshold in steady-state determination.

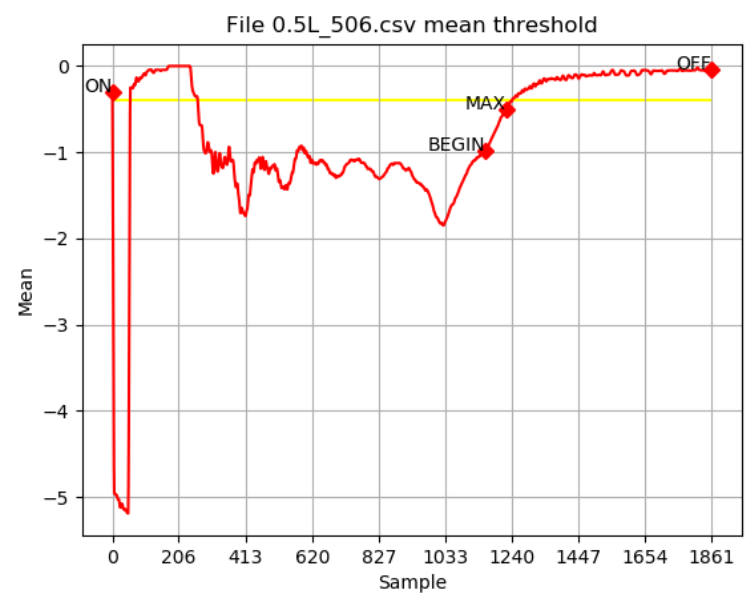

Fig. 6. Time and level (yellow line) thresholds for the water boiling process.

The existence of additional noise, which is highlighted in Fig. 6, demands the presence of these thresholds. At the top of the curve, it is apparent that the function decreases and then increases to a value of 0.1 . At the outset, therefore, after the curve decreases, we set a time threshold after which we wait until the curve crosses the level threshold.

\section{Results}

A series of experiments was conducted including checking sensors with different time inertia on water volumes 0.5 liters, 1.0 liters, 1.5 liters, 1.7 liters with different starting temperatures: $50{ }^{\circ} \mathrm{C}, 60^{\circ} \mathrm{C}, 70^{\circ} \mathrm{C}, 80^{\circ} \mathrm{C}, 90^{\circ} \mathrm{C}$. Using the experimental data sets, verification and refinement of the method.

A series of experiments was conducted including checking sensors with different time inertia (Response time) on water volumes 0.5 liters, 1.0 liters, 1.5 liters, 1.7 liters with 
different starting temperatures: $50^{\circ} \mathrm{C}, 60^{\circ} \mathrm{C}, 70^{\circ} \mathrm{C}, 80^{\circ} \mathrm{C}, 90^{\circ} \mathrm{C}$. Using the experimental data sets, we performed verification and refinement of the method.

For experiments to determine the point of initial boiling point, it should be clarified the boiling stages considered in the experiments. For the experiments, two stages were distinguished from the beginning of the heating of the volume of water to the moment of boiling. After reaching a certain temperature, the water enters the stage of active boiling. We mean active boiling as the process when the air filling the entire volume at the certain temperature, while the surface temperature has not reached the boiling point. The time required for active boiling depends on the volume of liquid.

In addition, the results of the refinement of the method and the obtained values of the thresholds were tested experimentally on the volumes of water $0.51,1.01,1.51,1.71$. In the experimental verification of the algorithm, the accuracy of determining the boiling point in small volumes is higher than in large volumes.

As mentioned previously, we set time and level thresholds for our algorithm. This enables resolving problems with additional undesirable noise. We also considered the case when the water was already heated, and the temperature of the water was reducing to below the boiling point. If we start the heating process again, our approach cannot determine the beginning of the steady-state. For this reason, we had to add the condition of waiting for the temperature, which is equal to approximately $80^{\circ} \mathrm{C}$.

In Fig. 7, the finite state machine is shown. Here, we are waiting for the water to reach the $80{ }^{\circ} \mathrm{C}$ point. Then, the moment when the curve is decreasing is tracked to the level threshold of -0.1 and falls below the zero points in the graph. Subsequently, we wait while the curve reaches the 0.1 point during 10 samples.

The algorithm accumulates the ten samples. This was added in order to exclude onetime bursts. Also, with a large volume of water, the process may not be so clearly determined on the graph and, despite the filtering of noise, for a short time, the value of the curve may reach certain conditions (bursts).

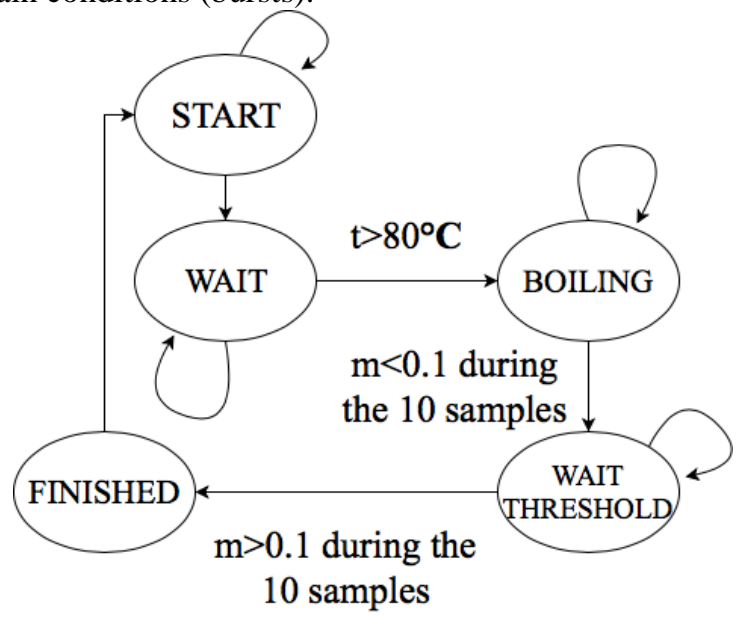

Fig. 7. Finite-state machine

The effect of application our approach is shown in Fig. 8. 


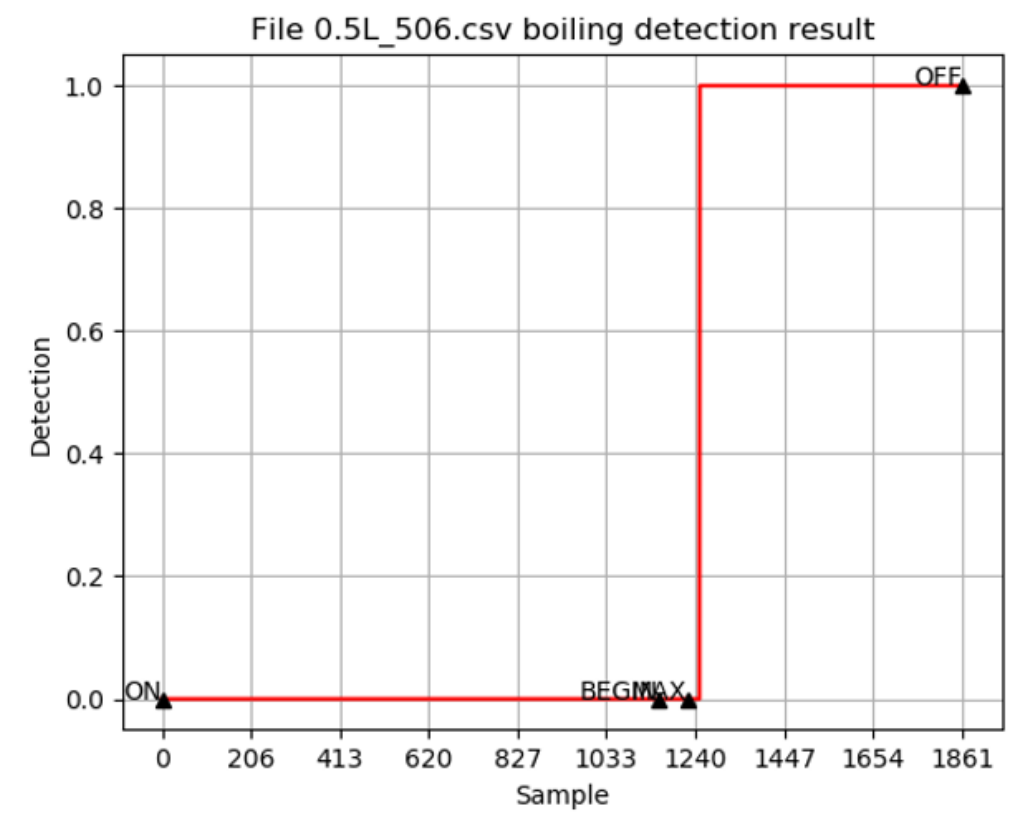

Fig. 8. The determination of the beginning the steady-state of the boiling process

\section{Discussion}

Having considered all related aspects for the accurate determination of the beginning the steady-state of a physical process such as a boiling process or other phase transition in a control system with limited resources, we assume our solution could be applied throughout general industrial applications.

This method has the advantage that it does not fully depend on absolute values. In the case of most experiments, the result was satisfactory, which indicates the applicability of the algorithm.

The delay which is added to the moment of determining the beginning of the steadystate process could be caused by the filtering part of the algorithm, which is there to remove undesirable noise. This was one of the main concerns faced during our study.

However, the particular results have shown the need to improve our approach further, because the water boiling process provided unstable results.

Subsequent improvements of this algorithm will consist of the improvement of the algorithmic part of noise reduction because noise reduction adds an additional time delay in the performing process of the algorithm.

The authors wish to acknowledge continuing support from Ready for Sky LLP, which deals with IoT in the Home Appliances development area and creates business solutions aimed at providing "smart" space to all homes.

The authors express thanks to all the current and past colleagues for their varied contributions to this research.

\section{References}

1. A. Sorokin, A.P. Shepeta., Smart Sens., Actuators, and MEMS VIII, (2017) 
2. A. Sorokin, A. Shepeta, M. Wattimena, Journal of Physics: Conf. Series, 1008 (2018)

3. J. Aaron, J.S. Bestley, V. Amalarani., IJPT, 7(2) (2015)

4. M.K. Bhanarkar, Ayyaz Ismail Nadaf, Prashant Prabhakar Shah., Int. J. of Dev. Research, 6 (2016)

5. Cuihui Shen,Yamin Zhao, Yiqun Li, Thermal Science and Eng. (2018)

6. J. Paul Guyer, P.E., R.A. Introd. to Boiler Control Systems. Continuing Edu. and Dev., Inc. Course No: E03-017

7. Appendix 1: Property Tables and Charts (SI Units), Scroll down to Table A-5 and read the temperature value of $99.61{ }^{\circ} \mathrm{C}$ at a pressure of $100 \mathrm{kPa}(1 \mathrm{bar})$. Obtained from McGraw-Hill's Higher Education website.

8. I. Seilonen, T. Pirttioja, K. Koskinen, Eng. Appl. Artif. Intell., 22, 1056-1067 (2009)

9. C. Sünder, A. Zoitl, C. Dutzler, Manuf. Research, 1(4), 405-420 (2006).

10. V. Venkatasubramanian, R. Rengaswamy, S.N. Kavuri, Comp. \& Chemical Eng., 27, 313-326 (2003)

11. C.K. Yoo, J.M. Lee, P.A. Vanrolleghem, I.B. Lee, Chemometrics and Intelligent Lab. Syst., 71, 151-163 (2004)

12. State-based approach of sugar boiling process automation in batch vacuum pan. Available from: https://www.researchgate.net/publication/311303189_State based_approach_of_sugar_boiling_process_automation_in_batch_vacuum_pan [accessed Aug 27 2018].

13. N. Bogach, V. Dyachkov, A. Lamtev, Y. Lezhenin, Eng. for Rural Develop., 16, 742747 (2017)

14. A.Y. Dnestrovskij, B.V. Kuteev, A.S. Bykov, A.A. Ivanov, V.E. Lukash, S.Y. Medvedev, V.Y. Sergeev, D.Y. Sychugov, R.R. Khayrutdinov, Nuclear Fusion, 55(6), (2015)

15. A.M. Belostotsky, P.A. Akimov, O.A. Negrozov, N.O. Petryashev, S.O. Petryashev, S.V. Sherbina, D.K. Kalichava, T.B. Kaytukov, Mag. Civ. Eng., 78(2), 169-178 (2018)

16. B.M. Medvedev, S.A. Molodyakov, S.M. Ustinov, S.A. Fyodorov, Int. Symp. Consum. Technol., ISCT, 66-69 (2018)

17. E. Fateeva, V. Badenko, A. Fedotov, I. Kochetkov, MATEC Web Conf., 170, 03033 (2018)

18. A.N. Evgrafov, V.I. Karazin, A.V. Khisamov, Int. Rev. Mech. Eng., 12(5) (2018)

19. S.Y. Galushin, Russ. Electr. Eng. 88 (12) (2017)

20. A. Bolotin, V. Bakayev, Journal of Physical Therapy Science (2017) 